\title{
Golden Mean and Fractal Dimension of Goose Down
}

\author{
Jing Gao ${ }^{a^{*}}$, Ning Pan ${ }^{b}$, Weidong Yu ${ }^{a}$ \\ a Textile College, Donghua University, West Yan'an Road 1882, Shanghai 200051, China \\ ${ }^{b}$ Biological and Agricultural Engineerings Department, University of California, Davis. \\ California 95616. U.S.A. \\ *Corresponding author, gao2001jing@mail.dhu.edu.cn
}

\begin{abstract}
Goose down is widely used in textile thermal products. The fractal dimension of goose down is studied in this paper both theoretically and experimentally, revealing its value to be close to the Golden Mean, 1.618. This near optimal fractal dimension may be attributable for the excellent thermal insulation and breath-ability of goose down. The potential applications of such a fractal structure are discussed as well.
\end{abstract}

Keywords: Golden mean, Fractal, Fractal dimension, Goose down

\section{Introduction}

As shown in our previous study [1], the SEM figures of goose down (see Figure1) exhibit an evident "self-similarity" between the parts of down fibers and the whole.

In simulating the morphologic structure and structural parameters of the down, a computer generated fractal graphics by using the Basal generating unit in Fig.1(d) for three hierarch levels is showed in Figure 2, and its fractal dimension is

$$
D_{f}=\frac{\ln N}{\ln L}=\frac{\ln 10}{\ln 4}=1.66
$$

Which is close to the Golden mean 1.618 [1].

It is well known that the golden mean has been found in many natural phenomena and played very significant roles [2-9]. It is both surprising and understandable that after many generations of evolution, goose down has acquired such an interesting fractal dimensional structure. The significance of the $D_{f}$ value for gooses' survival is quite self-explanatory. Because the possible range for the fractal dimension values is $0 \leqslant D_{f} \leqslant 2$ so that two extreme conditions will be: 1) $D_{f}=0$, that means there is no branches in goose down and the goose could not survive during the winter; 2) $D_{f}$ $=2$, that means the goose is covered air tightly by the down branches and little skin breath is allowed, another hardship too severer for goose to stand, especially in summer.

\section{Fractal confirmation based on the image of goose down}

We will demonstrate in this section the procedures to reach Eq.1. We select Figure1(c) as initial structural unit for analysis. For best imaging process effect, we need to determine a critical pixel level $p_{c r}$ which enable us to differentiate the actual structural information from the background noise. In other words the critical pixels level $p_{c r}$ assures a closest rendering of the actual picture into a computer 
image with the least distortion.

Since it is a monochromic case so that we use the grey level to regenerate the image. By choosing the background as complete dark and the goose down as total white. Figure 3 provides the information between the pixel numbers versus the grey level from the initial image captured. It is not difficult to discern that from left to right of the figure, as the grey level changes from darker to whiter, the corresponding pixel number reduces. It is at the pixel number $p=178$ where the profile shows a turning point; that is by choosing $p_{c r}=178$, the contrast between the image and background reaches the highest.

Then by using a two-value algorithm based on the threshold $p_{c r}=178$, we can turning all the pixel values into a binary system of black and white:

$$
\begin{aligned}
& p ? p_{c r}, \text { grey level }=1, \text { black } \\
& p<p_{c r}, \text { grey level }=0, \text { white }
\end{aligned}
$$

A goose down image thus enhanced is seen in Figure 4.

Introduce the Sandbox methods [10] to analyze the enhanced microscope image. For various areas (black or white), calculate the square box numbers $(N)$ at a given size $(L)$. Then the logarithm of $N$ and $L$ defines the fractal dimension value $D_{f}$, i.e.,

$$
D_{f}=\frac{\ln N}{\ln L}
$$

By assigning different $\mathrm{L}$ values and calculate the $\mathrm{N}$ value, we can plot a double logarithm curve as Figure 5. The curve of the double logarithm in Figure 5 has showed remarkable linearity with the linearity correlation coefficient achieves above 0.99, clearly revealing the prominent fractal characters of the goose down. Then the fractal dimension $D_{f}$ of the system can be derived though Eq.2.

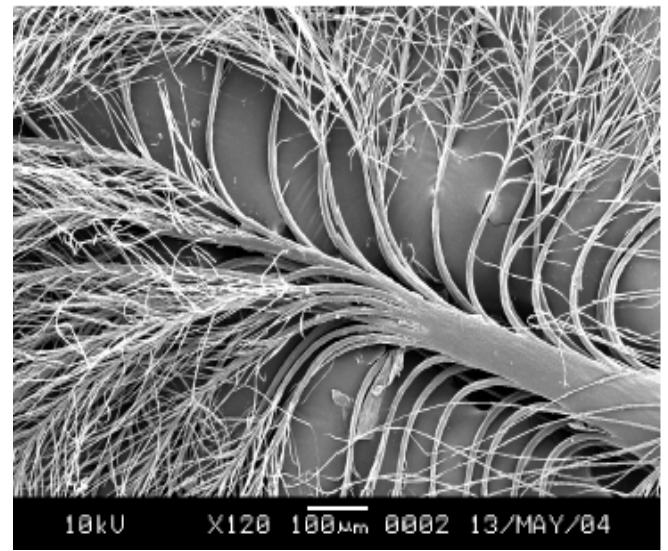

(a) Down branches diverging from down nucleus.

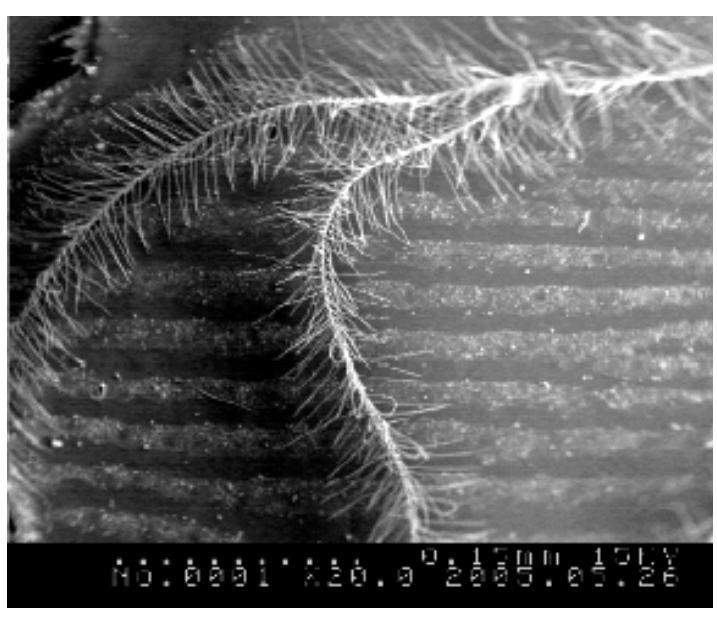

(b) Sub-branch diverging from main branch.

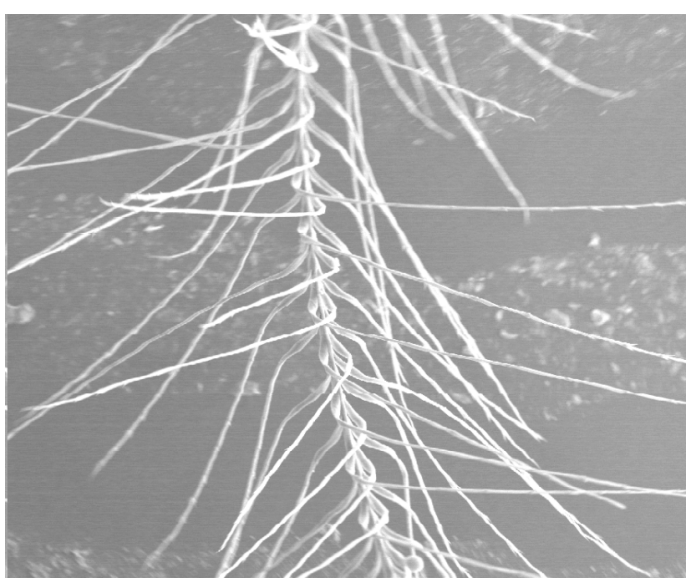

(c) Down fibrils diverging from sub-branch.

Fig. 1 (continued) 


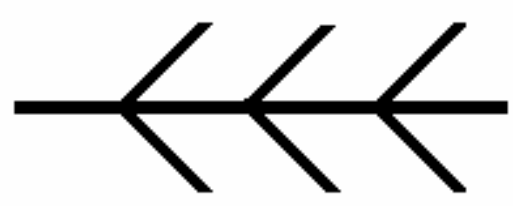

(d) The basal generating unit

Figure 1. Fractal structure and basal generating unit of goose down.

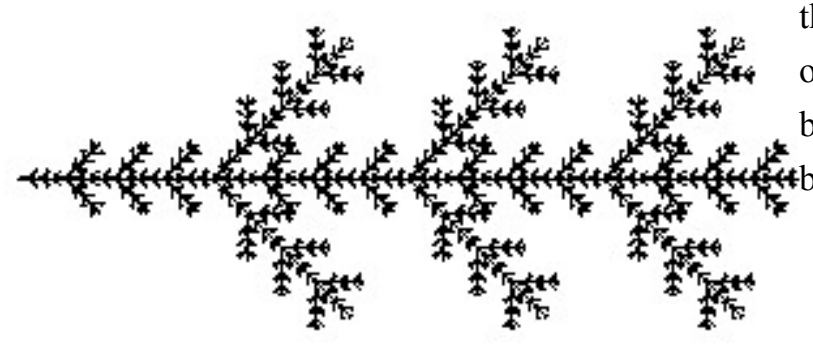

Figure 2. Computer generated fractal graphics at three levels of hierarchic iterations.

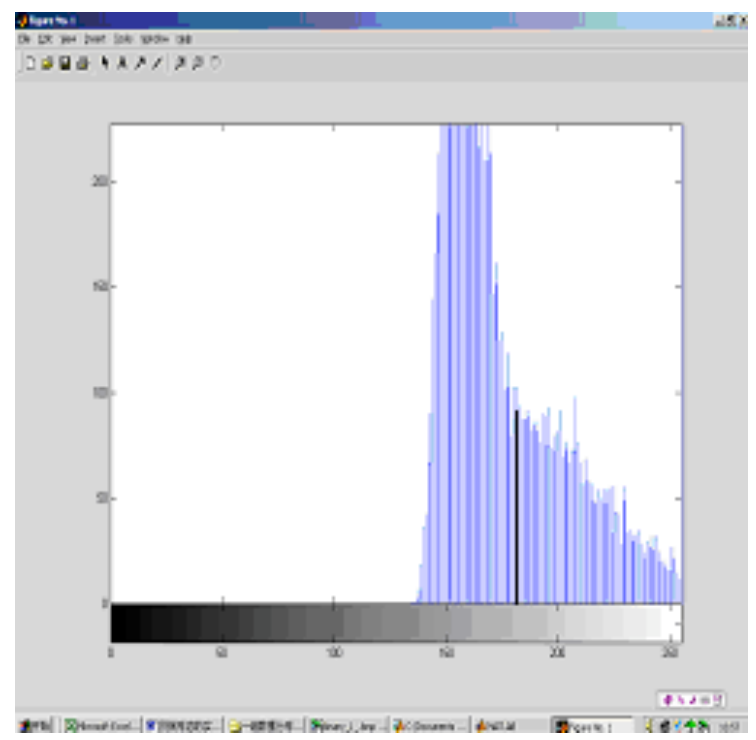

Figure 3.Selecting of the critical pixel.

\section{The actual fractal dimension of goose down}

Since this is an experimental approach with inevitable statistical error, at different measure scale, the corresponding fractal dimension $D_{f}$ will be slightly different. As an example using
Figure 4, we will show how this calculation is conducted, select the smallest branch diameter of the goose down as the basic unit measure scale $(l)$, and the side length of this figure is $l$, so the square box side

$$
L=\frac{l^{\prime}}{l}=\frac{515}{5}=103
$$

Using the area calculating procedure nothing that for each side the total pixels $=255$, we can obtain the areas occupied by the white pixels and black pixels respectively from Figure 4 to the black parts is $22.58 \%$, so

$$
100 \%: \frac{l^{\prime 2}}{l^{2}}=22.58 \%: N
$$

that is

$$
N=0.2258 \leftrightarrow 515^{2} / 5^{2}=2395.5122
$$

Thus the fractal dimension is

$$
D=\frac{\ln N}{\ln L}=\frac{\ln 2395.5122}{\ln 103}=1.678
$$

A statistical estimate based on adequate data can lead to the more accurate $D_{f}$ value.

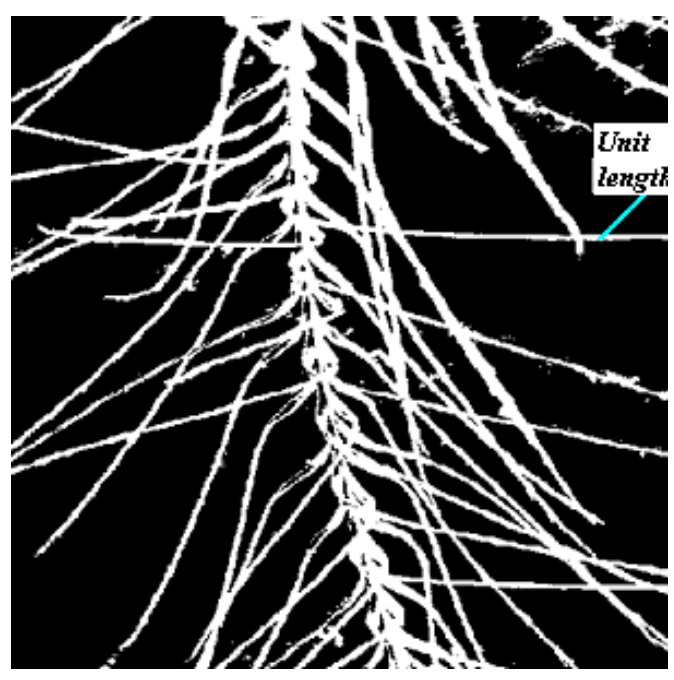

Figure 4.The picture after binary processing. 


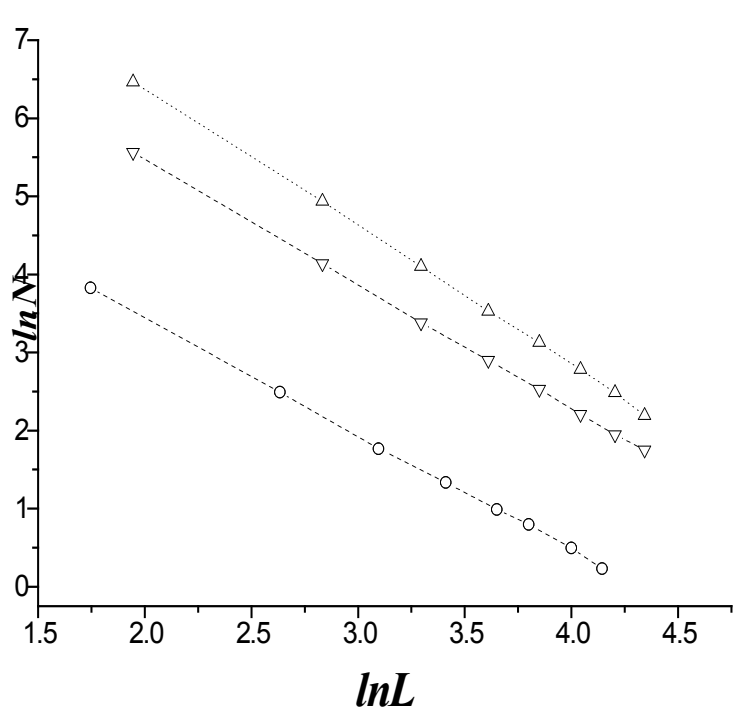

Figure 5. Double logarithm curve

\section{Conclusions}

In order to validate our previous analysis, we have used a computer image technique to actually measure the geometrical parameters to calculate the actual fractal dimension of the goose down.

The actual photo of a goose down unit is processed by a binary algorithms based on a chosen critical pixel level. Then an image analysis software is applied to acquire the geometrical data from an enhanced image, and the fractal dimension of the goose down branch is calculated as $D_{f}=1,678$, consistent with our previous theoretical prediction. Although a statistical estimate based on adequate data can lead to the more accurate $D_{f}$ value, our president result nonetheless confirms the inherent optimal structure of goose down, and this optimal morphologic structure must contribute greatly to the superior thermal insulating property of the goose down. The exact mechanisms associated however are still under investigation in our on going research.

\section{References}

[1] Gao Jing, Pan N, Yu WD. A fractal approach to goose down structure, International Journal of Nonlinear Sciences and Numerical Simulation. 7(1),113-116, 2006.

[2] Kuikka JT. Fractal analysis of organ structure, function and interactions, International Journal of Nonlinear Sciences and Numerical Simulation 7(3): 239-243, 2006

[3] Kuikka JT. Fractal analysis of day-to-day variation of heart rate and blood pressure: A case report, International Journal of Nonlinear Sciences and Numerical Simulation 6(2): 107-112, 2005

[4] Marek-Crnjac L. The golden mean in the topology of four-manifolds, in conformal field theory and in Cantorian space-time, Chaos, Solitons and Fractals, 28(5), 1113-1118 JUN 2006.

[5] M.S. El Naschie. Hilbert space, Poincare dodecahedron and golden mean transfiniteness, Chaos, Solitons and Fractals,vol.31,4,February 2007, 787-793.

[6] M.S. El Naschie. The Fibonacci code behind super strings and P-Branes. An answer to M.Kaku's fundamental question, Chaos, Solitons and Fractals, vol.31,3, February 2007,537-547.

[7] Cristina Stan, C.P.Cristescu and M. Agop. Golden mean relevance for chaos inhibition in a system of two coupled modified van der Poloscillators, Chaos, Solitons and Fractals, vol.31,4, February 2007, 1035-1040.

[8] Leonardo Di G. Sigalotti and Antonio Mejias. The golden ratio in special relativity, Chaos, Solitons and Fractals, vol.30,4, November 2006, 521-524.

[9] Alexey Stakhov. The golden section, secrets of the Egyptian civilization and harmony mathematics, Chaos, Solitons and Fractals, vol.30,2, October 2006, 490-505.

[10] Mandelbrot B. The Fractal Geometry of Nature, W. H. Freeman, New York, 1975. 